\title{
Constructing a Brownian Sheet with Values in a Compact Riemannian Manifold
}

\author{
É. Yu. Shamarova
}

Received March 5, 2004

Key words: Brownian motion, Brownian sheet, Laplace operator, compact Riemannian manifold, compact Lie group, cylindrical function.

In this paper, we propose a new method of constructing a two-parameter random field $\mathbf{W}_{M}^{x}(s, t)$, $x \in M$, with values in a compact Riemannian manifold $M$ possessing the property that the random processes $\mathbf{W}_{M}^{x}(\cdot, t)$ and $\mathbf{W}_{M}^{x}(s, \cdot)$ are Brownian motions on the manifold $M$ with parameters $t$ and $s$, respectively, issuing from the point $x$. (By a Brownian motion on a manifold $M$ with parameter $t$ we mean the diffusion process generated by the operator $-(t / 2) \Delta_{M}$, where $\Delta_{M}$ is the Laplace operator on the manifold $M$.) For the case in which the manifold is a compact Lie group, the two-parameter random field constructed in the paper coincides with the Brownian sheet defined by Malliavin [1] in 1991. (Malliavin called this random field a Brownian motion with values in $C([0,1], M)$, which is the set of continuous functions defined on the closed interval $[0,1]$ and taking values in $M$.) Nevertheless, for the case in which the manifold is a compact Lie group, the method proposed in the present paper essentially differs from that used in Malliavin's paper.

\section{FIRST STEP IN THE CONSTRUCTION OF THE RANDOM FIELD $\mathbf{W}_{M}^{x}$}

Suppose that $M$ is a $d$-dimensional compact Riemannian manifold without boundary isometrically embedded in $\mathbb{R}^{m}$. By a Brownian sheet with values in $\mathbb{R}^{m}$ we mean the family of $m$ independent standard Brownian sheets. Suppose that $\mathbf{W}_{t, s}$ is an $n$-dimensional Brownian sheet. Consider $\mathbf{W}_{t, s}$ as a process taking values in the space $C\left([0,1], \mathbb{R}^{m}\right)$. We denote this process by the symbol $\mathbf{W}_{t}$. We introduce the following notation: if $E$ is a locally convex space, then $E^{t}$ denotes $C([0, t], E)$; if $y \in C\left([0,1], \mathbb{R}^{m}\right)$ is a continuous function, then $\mathbb{W}^{y}$ denotes the distribution of the process $\mathbf{W}_{t}^{y}=y+\mathbf{W}_{t}$. If $\psi \in C\left([0,1], \mathbb{R}^{m}\right)$, then we define the process $\left(\mathbf{W}_{\psi}^{y}\right)_{t}=\psi(t)+\mathbf{W}_{t}^{y}$. Suppose that $\widetilde{\mathbb{W}}_{\psi}^{y}$ is the distribution of this process and $\mathrm{E}_{y, \psi}$ is the expectation with respect to the measure $\widetilde{\mathbb{W}}_{\psi}^{y}$. Further, $U_{\varepsilon}(M)$ denotes the $\varepsilon$-neighborhood of the manifold $M$. We consider $\mathbf{W}_{\psi}^{y}$ for functions $y$ and $\psi$ satisfying the conditions: $y(0) \in M, \psi(0)=0$. The goal of this section is to prove the existence of a limit (given below) with respect to the family of bounded continuous cylindrical functions, where by a cylindrical function $C\left([0,1] \times[0,1], \mathbb{R}^{m}\right) \rightarrow \mathbb{R}$ we mean a function $f$ for which there exists a finite collection of points $\tau_{1}, \ldots, \tau_{n}, \xi_{1}, \ldots, \xi_{k}$ and a function $\tilde{f}: \mathbb{R}^{n k} \rightarrow \mathbb{R}$ such that

$$
f(\omega)=\tilde{f}\left(\omega\left(\tau_{1}, \xi_{1}\right), \omega\left(\tau_{1}, \xi_{2}\right), \ldots, \omega\left(\tau_{n}, \xi_{k}\right)\right) .
$$

This limit defines the measure $\widetilde{\mathbb{W}}_{M, \psi, s, t}^{y}$ :

$$
\int_{C\left([0, s], \mathbb{R}^{m}\right)^{t}} f(\omega) \widetilde{\mathbb{W}}_{M, \psi, s, t}^{y}(d \omega)=\lim _{\varepsilon \rightarrow 0} \frac{\mathrm{E}_{y, \psi}\left\{f(\omega) \mathbb{I}_{\left\{\left(\mathbf{W}_{\psi}^{y}\right)_{t}(s) \in U_{\varepsilon}(M)\right\}}\right\}}{\widetilde{\mathbb{W}}_{\psi}^{y}\left\{\left(\mathbf{W}_{\psi}^{y}\right)_{t}(s) \in U_{\varepsilon}(M)\right\}} .
$$


Before proving the existence of such a limit, we consider the process

$$
\left(\mathbf{W}_{\psi, s}^{z}\right)_{t}=\psi(t)+B_{t}^{s}
$$

where $\psi:[0,1] \rightarrow \mathbb{R}^{m}$ is a continuous function satisfying the condition $\psi(0)=0$ and $B_{t}^{s}$ is a Brownian motion with parameter $s$ issuing from the point $z$. The results obtained for this process will be used for a subsequent construction.

Some results for the process $\left(\mathbf{W}_{\psi, s}^{z}\right)_{t}$. Suppose that $\mathbb{W}_{\psi, s}^{z}$ denotes the distribution of the process $\left(\mathbf{W}_{\psi, s}^{z}\right)_{t}$ and $\mathbf{E}_{z, \psi, s}$ is the expectation with respect to this distribution.

Lemma 1. The limit

$$
\int_{C\left([0, t], \mathbb{R}^{m}\right)} f(\omega) \mathbb{W}_{M, \psi, s, t}^{z}(d \omega)=\lim _{\varepsilon \rightarrow 0} \frac{\mathrm{E}_{z, \psi, s}\left\{f(\omega) \mathbb{I}_{\left\{\left(\mathbf{W}_{\psi, s}^{z}\right)_{t} \in U_{\varepsilon}(M)\right\}}\right\}}{\mathbb{W}_{\psi, s}^{z}\left\{\left(\mathbf{W}_{\psi, s}^{z}\right)_{t} \in U_{\varepsilon}(M)\right\}}
$$

with respect to the family of continuous bounded cylindrical functions, exists and defines the measure $\mathbb{W}_{M, \psi, s, t}^{z}$ in the integral on the left.

Sketch of the proof. Let us find a function $\tilde{f}: \mathbb{R}^{k+1} \rightarrow \mathbb{R}$ and a finite set of points $\tau_{1}, \ldots, \tau_{k}$ such that

$$
f(\omega)=\widetilde{f}\left(\omega\left(\tau_{1}\right), \ldots, \omega\left(\tau_{k}\right), \omega(t)\right)
$$

We have

$$
\begin{gathered}
\int_{C\left([0, t], \mathbb{R}^{m}\right)} f(\omega) \mathbb{W}_{M, \psi, s, t}^{z}(d \omega)=\lim _{\varepsilon \rightarrow 0} \frac{\int_{C\left([0, t], \mathbb{R}^{m}\right)} f(\omega) \mathbb{I}_{\left\{\omega: \omega(t) \in U_{\varepsilon}(M)\right\}} \mathbb{W}_{\psi, s}^{z}(d \omega)}{\mathbb{W}_{\psi, s}^{z}\left\{\omega: \omega(t) \in U_{\varepsilon}(M)\right\}} \\
=\lim _{\varepsilon \rightarrow 0} \frac{1}{\mathrm{P}^{\mathbb{W}}\left(t, 0, U_{\varepsilon}(M-z-\psi(t))\right)} \int_{\mathbb{R}^{m}} \mathrm{P}^{\mathbb{W}}\left(\tau_{1}, 0, d x_{1}\right) \int_{\mathbb{R}^{m}} \mathrm{P}^{\mathbb{W}}\left(\tau_{2}-\tau_{1}, x_{1}, d x_{2}\right) \cdots \\
\times \int_{U_{\varepsilon}(M-\psi(t)-z)} \mathrm{P}^{\mathbb{W}}\left(t-\tau_{k}, x_{k}, d x_{k+1}\right) \\
\times \widetilde{f}\left(x_{1}+z+\psi\left(\tau_{1}\right), \ldots, x_{k}+z+\psi\left(\tau_{k}\right), x_{k+1}+z+\psi(t)\right),
\end{gathered}
$$

where

$$
\mathrm{P}^{\mathbb{W}}(\tau, x, d z)=\frac{1}{(2 \pi s \tau)^{m / 2}} \exp \left\{-\frac{|z-x|^{2}}{2 s \tau}\right\} d z .
$$

Since the function in the integrand is bounded, it suffices, by Lebesgue's theorem, to prove that the following limit exists:

$$
\begin{aligned}
& \lim _{\varepsilon \rightarrow 0} \frac{\int_{\left(U_{\varepsilon}(M-\psi(t)-z)\right)} \tilde{f}\left(x_{1}+z+\psi\left(\tau_{1}\right), \ldots, x_{k+1}+z+\psi(t)\right) \mathrm{P}^{\mathbb{W}}\left(t-\tau_{k}, x_{k}, d x_{k+1}\right)}{\mathrm{P}^{\mathbb{W}}\left(t, 0, U_{\varepsilon}(M-z-\psi(t))\right)} \\
& =\lim _{\varepsilon \rightarrow 0} \frac{\int_{U_{\varepsilon}\left(M-\psi(t)-z-x_{k}\right)} \tilde{f}\left(x_{1}+z+\psi\left(\tau_{1}\right), \ldots, x_{k+1}+x_{k}+z+\psi(t)\right) \mathrm{P}^{\mathbb{W}}\left(t-\tau_{k}, 0, d x_{k+1}\right)}{\mathrm{P}^{\mathbb{W}}\left(t, 0, U_{\varepsilon}(M-z-\psi(t))\right.} .
\end{aligned}
$$

By $M_{1}$ we denote the manifold $M-\psi(t)-z-x_{k}$ and by $M_{2}$ the manifold $M-\psi(t)-z$. Further, suppose that

$$
\lambda_{\varepsilon}=\left.\frac{1}{\operatorname{vol}_{m-d}(\varepsilon)} l\right|_{U_{\varepsilon}\left(M_{1}\right)}, \quad \mu_{\varepsilon}=\left.\frac{1}{\operatorname{vol}_{m-d}(\varepsilon)} l\right|_{U_{\varepsilon}\left(M_{2}\right)},
$$


where $l$ is the Lebesgue measure on $\mathbb{R}^{m}$. We can easily see that the proof of the existence of this limit can be reduced to that of the existence of the limit

$$
\lim _{\varepsilon \rightarrow 0} \frac{\int_{\mathbb{R}^{m}} g\left(x_{k+1}\right) \exp \left\{-\frac{\left|x_{k+1}-x_{k}\right|^{2}}{2 s\left(t-\tau_{k}\right)}\right\} \lambda_{\varepsilon}\left(d x_{k+1}\right)}{\int_{\mathbb{R}^{m}} \exp \left\{-\frac{\left|x_{k+1}\right|^{2}}{2 s t}\right\} \mu_{\varepsilon}\left(d x_{k+1}\right)},
$$

where $g: \mathbb{R} \rightarrow \mathbb{R}$ is another symbol for the function $\tilde{f}$ introduced to indicate the dependence on the last variable solely. We can easily show that, as $\varepsilon \rightarrow 0$, the measures $\lambda_{\varepsilon}$ and $\mu_{\varepsilon}$ converge weakly to the surface measures on $M_{1}$ and $M_{2}$, respectively.

Lemma 2. The limit (1) with respect to the family of continuous bounded cylindrical functions exists.

Sketch of the proof. Suppose that $\mathrm{P}^{\widetilde{\mathbb{W}}}(t, y, \Gamma)=\widetilde{\mathbb{W}}^{y}(\omega: \omega(t) \in \Gamma)$ is the transition probability for the measure $\widetilde{\mathbb{W}}^{y}$, where $y \in C\left([0,1], \mathbb{R}^{m}\right)$. Further, suppose that the function

$$
\tilde{f}: C\left([0, s], \mathbb{R}^{m}\right)^{k+1} \rightarrow \mathbb{R}
$$

and the finite set of points $\tau_{1}, \tau_{2}, \ldots, \tau_{k}$ satisfy the relation

$$
f(\omega)=\widetilde{f}\left(\omega\left(\tau_{1}\right), \omega\left(\tau_{2}\right), \ldots, \omega\left(\tau_{k}\right), \omega(t)\right) .
$$

Let the symbol $\pi_{s}$ denote the coordinate mapping. The proof is carried out by using the following formula from [2, p. 204]:

$$
\begin{gathered}
\int_{C\left([0, s], \mathbb{R}^{m}\right)^{t}} f(\omega) \widetilde{\mathbb{W}}^{0}(d \omega)=\int_{C\left([0, s], \mathbb{R}^{m}\right)} \mathrm{P}^{\widetilde{\mathbb{W}}}\left(\tau_{1}, 0, d w_{1}\right) \int_{C\left([0, s], \mathbb{R}^{m}\right)} \mathrm{P}^{\widetilde{\mathbb{W}}}\left(\tau_{2}-\tau_{1}, w_{1}, d w_{2}\right) \cdots \\
\times \int_{\pi_{s}^{-1}\left(U_{\varepsilon}(M-\psi(t)-y(s))\right)} \widetilde{f}\left(w_{1}, \ldots, w_{k+1}\right) \mathrm{P}^{\widetilde{\mathbb{W}}}\left(t-\tau_{k}, w_{k}, d w_{k+1}\right)
\end{gathered}
$$

and applying Lemma 1 to the measure in the last integral.

\section{ASYMPTOTICS IN $t$ FOR AN INTEGRAL OF SPECIFIC FORM}

Proposition 1. Let $i$ be an isometric embedding of the manifold $M$ in $\mathbb{R}^{m}$ and $g \in C^{2}(M)$. Then

$\frac{1}{(2 \pi t)^{d / 2}} \int_{M} g(z) \exp \left\{-\frac{|z-y|^{2}}{2 t}\right\} \lambda_{M}(d z)=g(y)+\frac{t}{8} g(y)(c(y)-\operatorname{scal}(y))-\frac{t}{2} \Delta_{M} g(y)+t R(t, y)$,

where $|R(t, y)|<K t^{1 / 2}, K$ is a constant independent of $y, \operatorname{scal}(y)$ is the scalar curvature at the point $y$, and the function $c(y)$ is of the form

$$
c(y)=\sum_{k, l} \sum_{\alpha}\left(\frac{\partial^{2} i^{\alpha}}{\partial x^{k} \partial x^{l}}\right)^{2}(0),
$$

where the $x^{k}$ are the normal coordinates in a neighborhood $U_{y}$ of the point $y$ which are specified by the homeomorphism of the neighborhood $U_{y}$ onto a neighborhood of zero $U$ in $\mathbb{R}^{d}$. Independently of the local coordinates, $c(y)$ can be written as

$$
c(y)=-\left.\frac{1}{2} \Delta_{M} \Delta_{M}|y-\cdot|^{2}\right|_{y}-\frac{1}{3} \operatorname{scal}(y)
$$

and, therefore, $c(y)$ depends only on the embedding $i$. 
Sketch of the proof. We have obtained a more exact asymptotic expression in comparison with that obtained for an integral of similar form in [3]. The idea of the proof is the same.

Corollary 1. Suppose that $g \in C^{2}(M)$. Then the following asymptotics is valid:

$$
\frac{\int_{M} g(z) \exp \left\{-\frac{|z-y|^{2}}{2 t}\right\} \lambda_{M}(d z)}{\int_{M} \exp \left\{-\frac{|z-y|^{2}}{2 t}\right\} \lambda_{M}(d z)}=g(y)-\frac{t}{2} \Delta_{M} g(y)+t R_{1}(t, y),
$$

where $\left|R_{1}(t, y)\right|<K_{1} t^{1 / 2}$, and $K_{1}$ is a constant independent of $y$.

Corollary 2. Suppose that $g \in C^{2}(M), y \in M$, and $\psi$ is a Hölder function of Hölder order $\alpha$, $1 / 3<\alpha<1 / 2$, such that $\psi(0)=0$. Suppose that $\operatorname{Pr}_{M}$ is the projection mapping onto the manifold $M$ along the subspaces normal to the manifold and defined in a suitable neighborhood of the manifold $\psi_{M}(t, y)=\operatorname{Pr}_{M}(y+\psi(t))$. Then the following asymptotics is valid:

$$
\frac{\int_{M} g(z) \exp \left\{-\frac{|z-y-\psi(t)|^{2}}{2 t}\right\} \lambda_{M}(d z)}{\int_{M} \exp \left\{-\frac{|z-y-\psi(t)|^{2}}{2 t}\right\} \lambda_{M}(d z)}=g\left(y+\psi_{M}(t)\right)-\frac{t}{2} \Delta_{M} g(y)+t R_{2}(t, y),
$$

where $\left|R_{2}(t, y)\right|<K_{2} t^{3 \alpha-1}$ and $K_{2}$ is a constant.

\section{SECOND STEP IN THE CONSTRUCTION OF THE RANDOM FIELD $\mathbf{W}_{M}^{x}$}

Suppose that $f$ is a continuous bounded cylindrical function on $C\left([0, s], \mathbb{R}^{m}\right)^{1}$ and $\varphi: \mathbb{R} \rightarrow M$ is a function which is the trajectory of the Brownian motion on $M$ such that $\varphi(0)=x$. Suppose that $\mathcal{P}_{1}=\left\{0=t_{0} \leq t_{1} \leq \cdots \leq t_{n}=1\right\}$ is a partition of the interval $[0,1]$. If $E$ is a locally convex space, then to each $\omega \in E^{1}$ we can assign a finite sequence of $n$ elements

$$
\left(\omega_{1}, \omega_{2}, \ldots, \omega_{n}\right) \in E^{t_{1}} \times E^{t_{2}-t_{1}} \times \cdots \times E^{t_{n}-t_{n-1}},
$$

where $\omega_{j}$ is defined on the interval $\left[0, t_{j}-t_{j-1}\right]$ by the formula $\omega_{j}(t)=\omega\left(t_{j-1}+t\right)$. We define the function $\varphi_{t_{i-1} t_{i}}$ on the interval $\left[0, t_{i}-t_{i-1}\right]$ as follows:

$$
\varphi_{t_{i-1} t_{i}}(t)=\varphi\left(t_{i-1}+t\right)-\varphi\left(t_{i-1}\right) .
$$

Let us define the measure $\widetilde{\mathbb{W}}_{M, \varphi, s, \mathcal{P}_{1}}^{x}$ by the formula

$$
\begin{aligned}
& \int_{C\left([0, s], \mathbb{R}^{m}\right)^{1}} f(\omega) \widetilde{\mathbb{W}}_{M, \varphi, s, \mathcal{P}_{1}}^{x}(d \omega)=\int_{C\left([0, s], \mathbb{R}^{m}\right)^{t_{1}}} \widetilde{\mathbb{W}}_{M, \varphi_{0 t_{1}}, s, t_{1}}^{x}\left(d \omega_{1}\right) \\
& \quad \times \int_{C\left([0, s], \mathbb{R}^{m}\right)^{t_{2}-t_{1}}} \widetilde{\mathbb{W}}_{M, \varphi_{t_{1} t_{2}}, s, t_{2}-t_{1}}^{\omega_{1}\left(t_{1}\right)}\left(d \omega_{2}\right) \cdots \\
& \quad \times \int_{C\left([0, s], \mathbb{R}^{m}\right)^{t_{n}-t_{n-1}}} \widetilde{\mathbb{W}}_{M, \varphi_{t_{n-1} t_{n}, s, t_{n}-t_{n-1}}^{\omega_{n-1}\left(t_{n-1}-t_{n-2}\right)}}\left(d \omega_{n}\right) f\left(\omega_{1}, \omega_{2}, \ldots, \omega_{n}\right) .
\end{aligned}
$$

It is readily verified that $\omega_{i}\left(t_{i}-t_{i-1}\right)(0) \in M$, so that the measure $\widetilde{\mathbb{W}}_{M, \varphi, s, \mathcal{P}_{1}}^{x}$ is well defined. Further, let $\mathcal{P}_{2}=\left\{0=s_{0} \leq s_{1} \leq \cdots \leq s_{k}=1\right\}$ be a partition of the interval $[0,1]$. Now, suppose that $s$ is a time parameter. Instead of the symbol $\mathbb{W}_{M, \varphi, s, \mathcal{P}_{1}}^{x}$, we shall write $\mathbb{\mathbb { W }}_{M, s, \mathcal{P}_{1}}^{\varphi}$. Let us define the measure $\mathbb{W}_{M, \mathcal{P}_{1}, \mathcal{P}_{2}}^{x}$ by the formula

$$
\begin{aligned}
& \int_{C\left([0,1], \mathbb{R}^{m}\right)^{1}} f(\omega) \mathbb{W}_{M, \mathcal{P}_{1}, \mathcal{P}_{2}}^{x}(d \omega)=\int_{C\left([0,1], \mathbb{R}^{m}\right)^{s_{1}}} \widetilde{\mathbb{W}}_{M, s_{1}, \mathcal{P}_{1}}^{x}\left(d \omega_{1}\right) \\
& \quad \times \int_{C\left([0,1], \mathbb{R}^{m}\right)^{s_{2}-s_{1}}} \widetilde{\mathbb{W}}_{M, s_{2}-s_{1}, \mathcal{P}_{1}}^{\omega_{1}\left(s_{1}\right)}\left(d \omega_{2}\right) \cdots \\
& \quad \times \int_{C\left([0,1], \mathbb{R}^{m}\right)^{s_{n}-s_{n-1}}} \widetilde{\mathbb{W}}_{M, s_{n}-s_{n-1}, \mathcal{P}_{1}}^{\omega_{n-1}\left(s_{n-1}-s_{n-2}\right)}\left(d \omega_{n}\right) f\left(\omega_{1}, \ldots, \omega_{n}\right) .
\end{aligned}
$$


Theorem 1. For each $x \in M$, if the meshes of the partitions $\mathcal{P}_{1}$ and $\mathcal{P}_{2}$ tend to zero, then the sequence of measures $\mathbb{W}_{M, \mathcal{P}_{1}, \mathcal{P}_{2}}^{x}$ is weakly convergent to the measure $\mathbb{W}_{M}^{x}$ with respect to the family of continuous bounded cylindrical functions. The measure $\mathbb{W}_{M}^{x}$ regarded as the distribution of a process with values in $C([0,1], M)$, possesses a transition probability at time $t$ coinciding with the distribution of the Brownian motion with parameter $t$ on the manifold issuing from the point $x$.

Sketch of the proof. Suppose that

$$
\begin{aligned}
& h \cdot \mathbb{W}_{M, \varphi, s, \mathcal{P}_{1}}^{x}\left(=h \cdot \mathbb{W}_{M, s, \mathcal{P}_{1}}^{\varphi}\right)=\int_{C\left([0,1], \mathbb{R}^{m}\right)} h(\omega) \mathbb{W}_{M, \varphi, s, \mathcal{P}_{1}}^{x}(d \omega)
\end{aligned}
$$

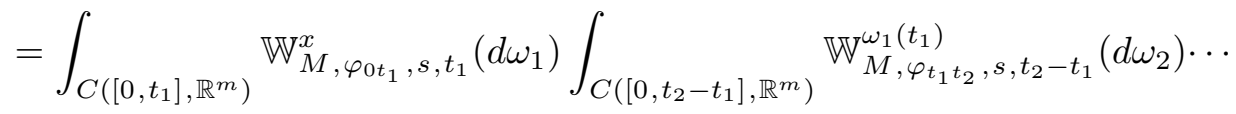

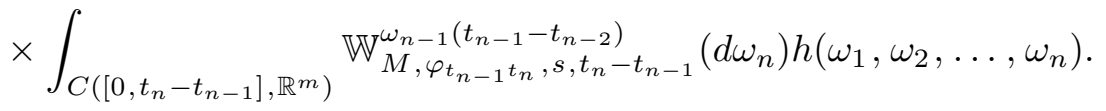

Suppose that there exists a function $\tilde{f}: C\left([0,1], \mathbb{R}^{m}\right) \rightarrow \mathbb{R}$ such that $f(\omega)=\tilde{f}(\omega(t))$. Then

$$
\begin{aligned}
\int_{C\left([0, s], \mathbb{R}^{m}\right)^{1}} f(\omega) \widetilde{\mathbb{W}}_{M, s, \mathcal{P}_{1}}^{\varphi}(d \omega) & =\int_{C\left([0,1], \mathbb{R}^{m}\right)} \tilde{f}(w) \widetilde{\mathbb{W}}_{M, s, \mathcal{P}_{1}}^{\varphi} \circ \pi_{s}^{-1}(d w) \\
& =\int_{C\left([0,1], \mathbb{R}^{m}\right)} \tilde{f}(w) \mathbb{W}_{M, s, \mathcal{P}_{1}}^{\varphi}(d w) .
\end{aligned}
$$

This yields

$$
\begin{aligned}
& \int_{C\left([0,1], \mathbb{R}^{m}\right)^{1}} f(\omega) \mathbb{W}_{M, \mathcal{P}_{1}, \mathcal{P}_{2}}^{x}(d \omega)=\int_{C\left([0,1], \mathbb{R}^{m}\right)} \mathbb{W}_{M, s_{1}, \mathcal{P}_{1}}^{x}\left(d w_{1}\right) \\
& \quad \times \int_{C\left([0,1], \mathbb{R}^{m}\right)} \mathbb{W}_{M, s_{2}-s_{1}, \mathcal{P}_{1}}^{w_{1}}\left(d w_{2}\right) \cdots \int_{C\left([0,1], \mathbb{R}^{m}\right)} \mathbb{W}_{M, s_{n-1}-s_{n-2}, \mathcal{P}_{1}}^{w_{n-2}}\left(d w_{n-1}\right) \\
& \quad \times \int_{C\left([0,1], \mathbb{R}^{m}\right)} \mathbb{W}_{M, s_{n}-s_{n-1}, \mathcal{P}_{1}}^{w_{n-1}}\left(d w_{n}\right) \widetilde{f}\left(w_{n}\right) .
\end{aligned}
$$

Consider the integral

$$
\int_{C\left([0, t], \mathbb{R}^{m}\right)} g(\omega) \mathbb{W}_{M, \psi, s, t}^{z}(d \omega)
$$

where the function $g \in C\left([0, t], \mathbb{R}^{m}\right)$ is such that there exists a function $\widetilde{g} \in C(\mathbb{R})$ for which $g(\omega)=\widetilde{g}(\omega(t))$. As a result of simple calculations, we obtain

$$
\begin{aligned}
\int_{C\left([0, t], \mathbb{R}^{m}\right)} g(\omega) \mathbb{W}_{M, \psi, s, t}^{z}(d \omega) & =\lim _{\varepsilon \rightarrow 0} \frac{\int_{C\left([0, t], \mathbb{R}^{m}\right)} g(\omega) \mathbb{I}_{\left\{\omega: \omega(t) \in U_{\varepsilon}(M)\right\}}(\omega) \mathbb{W}_{\psi, s}^{z}(d \omega)}{\mathbb{W}_{\psi, s}^{z}\left\{\omega: \omega(t) \in U_{\varepsilon}(M)\right\}} \\
& =\frac{\int_{M} \exp \left\{-\frac{\left|x_{1}-z-\psi(t)\right|^{2}}{2 t s}\right\} \widetilde{g}\left(x_{1}\right) \lambda_{M}\left(d x_{1}\right)}{\int_{M} \exp \left\{-\frac{\left|x_{1}-z-\psi(t)\right|^{2}}{2 t s}\right\} \lambda_{M}\left(d x_{1}\right)} .
\end{aligned}
$$

First, suppose that the function $f$ is such that there exists a function $p: \mathbb{R}^{m} \rightarrow \mathbb{R}$ and numbers $t, s \in[0,1]$ for which $f(\omega)=p(\omega(t, s))$. The integral

$$
\int_{C\left([0,1], \mathbb{R}^{m}\right)^{1}} f(\omega) \mathbb{W}_{M, \mathcal{P}_{1}, \mathcal{P}_{2}}^{x}(d \omega)
$$


is of the form

$$
\begin{aligned}
& \frac{\int_{M} \exp \left\{-\frac{\left|x_{1}-x\right|^{2}}{2 \Delta s_{1} \Delta t_{1}}\right\} d x_{1}}{\int_{M} \exp \left\{-\frac{\left|\bar{x}_{1}-x\right|^{2}}{2 \Delta s_{1} \Delta t_{1}}\right\} d \bar{x}_{1}} \cdots \frac{\int_{M} \exp \left\{-\frac{\left|x_{n-1}-x_{n-2}\right|^{2}}{2 \Delta s_{1} \Delta t_{n-1}}\right\} d x_{n-1}}{\int_{M} \exp \left\{-\frac{\left|\bar{x}_{n-1}-x_{n-2}\right|^{2}}{2 \Delta s_{1} \Delta t_{n-1}}\right\} d \bar{x}_{n-1}} \frac{\int_{M} \exp \left\{-\frac{\left|x_{n}-x_{n-1}\right|^{2}}{2 \Delta s_{1} \Delta t_{n}}\right\} d x_{n} \exp \left\{-\frac{\left|\bar{x}_{n}-x_{n-1}\right|^{2}}{2 \Delta s_{1} \Delta t_{n}}\right\} d \bar{x}_{n}}{\int_{M}} \\
& \times \frac{\int_{M} \exp \left\{-\frac{\left|y_{1}-x_{1}\right|^{2}}{2 \Delta s_{2} \Delta t_{1}}\right\} d y_{1}}{\int_{M} \exp \left\{-\frac{\left|\bar{y}_{1}-x_{1}\right|^{2}}{2 \Delta s_{2} \Delta t_{1}}\right\} d \bar{y}_{1}} \cdots \frac{\int_{M} \exp \left\{-\frac{\left|y_{n-1}-y_{n-2}-x_{n-1}+x_{n-2}\right|^{2}}{2 \Delta s_{2} \Delta t_{n-1}}\right\} d y_{n-1}}{\int_{M} \exp \left\{-\frac{\left|\bar{y}_{n-1}-y_{n-2}-x_{n-1}+x_{n-2}\right|^{2}}{2 \Delta s_{2} \Delta t_{n-1}}\right\} d \bar{y}_{n-1}} \\
& \times \frac{\int_{M} \exp \left\{-\frac{\left|y_{n}-y_{n-1}-x_{n}+x_{n-1}\right|^{2}}{2 \Delta s_{2} \Delta t_{n}}\right\} d y_{n}}{\int_{M} \exp \left\{-\frac{\left|\bar{y}_{n}-y_{n-1}-x_{n}+x_{n-1}\right|^{2}}{2 \Delta s_{2} \Delta t_{n}}\right\} d \bar{y}_{n}} \cdots \\
& \times \frac{\int_{M} \exp \left\{-\frac{\left|u_{1}-z_{1}\right|^{2}}{2 \Delta s_{k-1} \Delta t_{1}}\right\} d u_{1}}{\int_{M} \exp \left\{-\frac{\left|\bar{u}_{1}-z_{1}\right|^{2}}{2 \Delta s_{k-1} \Delta t_{1}}\right\} d \bar{u}_{1}} \cdots \frac{\int_{M} \exp \left\{-\frac{\left|u_{n-1}-u_{n-2}-z_{n-1}+z_{n-2}\right|^{2}}{2 \Delta s_{k-1} \Delta t_{n-1}}\right\} d u_{n-1}}{\int_{M} \exp \left\{-\frac{\left|\bar{u}_{n-1}-u_{n-2}-z_{n-1}+z_{n-2}\right|^{2}}{2 \Delta s_{k-1} \Delta t_{n-1}}\right\} d \bar{u}_{n-1}} \\
& \times \frac{\int_{M} \exp \left\{-\frac{\left|u_{n}-u_{n-1}-z_{n}+z_{n-1}\right|^{2}}{2 \Delta s_{k-1} \Delta t_{n}}\right\} d u_{n}}{\int_{M} \exp \left\{-\frac{\left|\bar{u}_{n}-u_{n-1}-z_{n}+z_{n-1}\right|^{2}}{2 \Delta s_{k-1} \Delta t_{n}}\right\} d \bar{u}_{n}} \\
& \times \frac{\int_{M} \exp \left\{-\frac{\left|v_{1}-u_{1}\right|^{2}}{2 \Delta s_{k} \Delta t_{1}}\right\} d v_{1}}{\int_{M} \exp \left\{-\frac{\left|\bar{v}_{1}-u_{1}\right|^{2}}{2 \Delta s_{k} \Delta t_{1}}\right\} d \bar{v}_{1}} \cdots \frac{\int_{M} \exp \left\{-\frac{\left|v_{n-1}-v_{n-2}-u_{n-1}+u_{n-2}\right|^{2}}{2 \Delta s_{k} \Delta t_{n-1}}\right\} d v_{n-1}}{\int_{M} \exp \left\{-\frac{\left|\bar{v}_{n-1}-v_{n-2}-u_{n-1}+u_{n-2}\right|^{2}}{2 \Delta s_{k} \Delta t_{n-1}}\right\} d \bar{v}_{n-1}} \\
& \times \frac{\int_{M} \exp \left\{-\frac{\left|v_{n}-v_{n-1}-u_{n}+u_{n-1}\right|^{2}}{2 \Delta s_{k} \Delta t_{n}}\right\} p\left(v_{n}\right) d v_{n}}{\int_{M} \exp \left\{-\frac{\left|\bar{v}_{n}-v_{n-1}-u_{n}+u_{n-1}\right|^{2}}{2 \Delta s_{k} \Delta t_{n}}\right\} d \bar{v}_{n}},
\end{aligned}
$$

where $\Delta t_{i}=t_{i}-t_{i-1}, \Delta s_{j}=s_{j}-s_{j-1}$, and, to simplify the notation, instead of $\lambda_{M}(d z)$ we use $d z$. We have also assumed that $t_{n}=t$ and $s_{k}=s$. Let us denote this integral by $I\left(\mathcal{P}_{1}, \mathcal{P}_{2}, p\right)$.

Lemma 3. The integral $I\left(\mathcal{P}_{1}, \mathcal{P}_{2}, p\right)$ converges to $\exp \left\{-\frac{s t}{2} \Delta_{M}\right\} p$ if the meshes of $\left|\mathcal{P}_{1}\right|$ and $\left|\mathcal{P}_{2}\right|$ tend to zero.

Sketch of the proof. Using Corollaries 1 and 2 of Proposition 1, we obtain the following asymptotics for the integral $I\left(\mathcal{P}_{1}, \mathcal{P}_{2}, p\right)$ :

$$
I\left(\mathcal{P}_{1}, \mathcal{P}_{2}, p\right)(x)=p(x)-\frac{s t}{2} \Delta_{M} p(x)+O\left(s^{2} t^{2}\right)+O\left(\left|\mathcal{P}_{2}\right|,\left|\mathcal{P}_{1}\right|\right)
$$

Hence we see that the following limit exists:

$$
\lim _{\left|\mathcal{P}_{1}\right| \rightarrow 0,\left|\mathcal{P}_{2}\right| \rightarrow 0} I\left(\mathcal{P}_{1}, \mathcal{P}_{2}, p\right)(x)=p(x)-\frac{s t}{2} \Delta_{M} p(x)+O\left(s^{2} t^{2}\right)\left(=\left(Q_{s t} p\right)(x)\right) .
$$

Further, we verify that $Q_{\tau+\Delta \tau}=Q_{\tau} Q_{\Delta \tau}$. Let $s$ and $t$ satisfy $\tau=s t$. Let us find a $\Delta s$ and $\Delta t$ such that $\tau+\Delta \tau=(s+\Delta s)(t+\Delta t)$, and consider the integral $I\left(\mathcal{P}_{1}^{[0, t+\Delta t]}, \mathcal{P}_{2}^{[0, s+\Delta s]}, p\right)$ for partitions of the closed intervals $[0, s+\Delta s]$ and $[0, t+\Delta t]$ resulting from supplementing the corresponding partitions of the closed intervals $[0, s]$ and $[0, t]$ by points of partitions of the closed intervals $[t, t+\Delta t],[s, s+\Delta s]$. We can easily see from the structure of the integral $I\left(\mathcal{P}_{1}, \mathcal{P}_{2}, p\right)$ that

$$
I\left(\mathcal{P}_{1}^{[0, t+\Delta t]}, \mathcal{P}_{2}^{[0, s+\Delta s]}, p\right)=I\left(\mathcal{P}_{1}^{[0, t]}, \mathcal{P}_{2}^{[0, s]}, I\left(\mathcal{P}_{1}^{[t, t+\Delta t]}, \mathcal{P}_{2}^{[s, s+\Delta s]}, p\right)\right)
$$


Passing to the limit in this expression and taking $\Delta \tau=s \Delta t+t \Delta s+\Delta s \Delta t$ into account, we find that $Q_{\tau}$ is a semigroup which, by the proof above, satisfies

$$
\left(Q_{\tau} p\right)(x)=p(x)-\frac{\tau}{2} \Delta_{M} p(x)+O\left(\tau^{2}\right)
$$

Thus, we find that

$$
Q_{\tau}=\exp \left\{-\frac{\tau}{2} \Delta_{M}\right\}
$$

For a function $f$ depending on $\omega$ at several points, such as at points $\xi_{i} \in[0, s]$ and $\tau_{j} \in[0, t]$, the integral $I\left(\mathcal{P}_{1}, \mathcal{P}_{2}, p\right)$ will be of the same form. The convergence examined above occurs on each square $\left[\xi_{i-1}, \xi_{i}\right] \times\left[\tau_{j-1}, \tau_{j}\right]$. Each of operators of the form

$$
\exp \left\{-\frac{\Delta \xi_{i} \Delta \tau_{j}}{2} \Delta_{M}\right\}
$$

acts on the corresponding variable of the function $p$ defined as

$$
f(\omega)=p\left(\omega_{11}\left(\xi_{1}, \tau_{1}\right), \ldots, \omega_{k l}\left(\xi_{l}, \tau_{l}\right)\right),
$$

where $\omega_{i j}$ is defined on $\left[0, \xi_{i}-\xi_{i-1}\right] \times\left[0, \tau_{j}-\tau_{j-1}\right]$ by the formula

$$
\omega_{i j}(s, t)=\omega\left(\xi_{i-1}+s, \tau_{j-1}+t\right) .
$$

Corollary 3. Suppose that $M$ is a compact Lie group. Then the random field $\mathbf{W}_{M}^{x}$ regarded as a process with values in $C([0,1], M)$, coincides with the Brownian motion constructed in [1].

Sketch of the proof. The proof follows from Theorem 1 and Theorem 2.15 from [4] (Theorem 2.15 from [4] was also proved in Lemma 3.3 from [5]).

\section{REFERENCES}

1. P. Malliavin, in: Diffusion Processes and Related Problems in Analysis (Evanston, IL, 1989), vol. 1, Birkhäuser, Boston, MA, 1990, pp. 17-31.

2. N. Ikeda and Sh. Watanabe, Stochastic Differential Equations and Diffusion Processes, North-Holland, Amsterdam, 1981.

3. O. G. Smolyanov and H. von Weizsäcker, and O. Wittich, Canad. Math. Soc. Conference Proc., 29 (2000), 589-602.

4. B. K. Driver and V. K. Srimurthy, Ann. Probab., 29 (2001), no. 2, 691-723.

5. V. K. Srimurthy, Probab. Theory Related Fields, 118 (2000), no. 4, 522-546.

M. V. Lomonosov Moscow State University 